\title{
Efetividade tecnológica na prontidão para mudança comportamental em hipertensão e excesso ponderal
}

Technological effectiveness in readiness for behavior change in hypertension and overweight Efectividad tecnológica en la prontitud para cambios de comportamiento en hipertensión y exceso ponderal

Jênifa Cavalcante dos Santos Santiago ${ }^{1}$ (i) https://orcid.org/0000-0001-9815-8698

Thereza Maria Magalhães Moreira' ${ }^{1}$ ic htps://orcid.org/0000-0003-1424-0649,

Raquel Sampaio Florêncio ${ }^{1}$ ic https://orcid.org/0000-0003-3119-7187

José Wicto Pereira Borges ${ }^{1}$ if hitps://orcid.org/0000-0002-3292-1942

Vera Lúcia Mendes de Paula Pessoa ${ }^{1}$ en nttps://orcid.org/0000-0002-8158-7071

Ana Célia Caetano de Souza ${ }^{1}$ io nttps://orcid.org/0000-0001-9480-7195

Santiago JC, Moreira TM, Florêncio citar: JW, Pessoa VL, Souza AC. Efetividade tecnológica na prontidão para mudança comportamental em hipertensão e excesso ponderal. Acta Paul Enferm. 2021;34:eAPE00395.

DOI http://dx.doi.org/10.37689/actaape/2021A000395

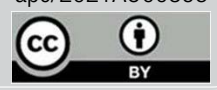

Descritores Hipertensão; Peso corporal; Tecnologia educacional

Keywords Hypertension; Body weight; Educational technology

Descriptores Hipertensión; Peso corporal; Tecnologia educacional

Submetido 29 de Fevereiro de 2020

Aceito 2 de Dezembro de 2020

Autor correspondente

Raquel Sampaio Florêncio E-mail: raquelsampy@hotmail.com

\section{Resumo}

Objetivo: Testar a efetividade de uma tecnologia educativa sobre excesso ponderal no estágio de prontidão para mudança de comportamento do adulto com hipertensão arterial sistêmica e excesso ponderal.

Métodos: Estudo quase-experimental, com dois grupos, comparação e intervenção, cada um com 36 participantes, cujo estágio de prontidão para mudança de comportamento foi avaliado antes (momento zero) e depois (momento um) da intervenção (implementação ou não da cartilha), após um mês (momento dois) e dois meses (momento três) do primeiro contato, via telefone. 0 estágio de prontidão para mudança de comportamento foi comparado antes e depois e entre os grupos pelo teste t de Student para amostra em pares e para grupos independentes, respectivamente.

Resultados: Os resultados mostraram que a implementação da cartilha favorece pensamentos de mudança mais duradouros, com diferenças estatisticamente significativas entre os grupos no momento dois $(p=0,020)$ e três $(p=0,003)$ e entre os momentos zero e um $(p<0,0001)$, momentos zero e dois ( $p=0,001)$, momentos zero e três $(p<0,0001)$ e momento dois e três $(p<0,0001)$ no grupo intervenção.

Conclusão: A cartilha educativa foi, portanto, validada e favoreceu o avanço no estágio de prontidão para mudança de comportamento, mostrando-se efetiva para práticas educativas em saúde. A validação da cartilha permite sua utilização em atividades de educação em saúde de modo confiável no público em questão.

\section{Abstract}

Objective: To test the effectiveness of an educational technology on overweight in the stage of readiness for behavior change in adults with hypertension and overweight.

Methods: This is a quasi-experimental study, with two groups, comparison and intervention, each with 36 participants, whose stage of readiness for behavior change was assessed before (moment zero) and after (moment one) of the intervention (implementation or not) of the booklet after one month (moment two) and two months (moment three) of the first contact via telephone. The stage of readiness for behavior change was compared before and after and between groups by Student's t test for sample in pairs and for independent groups, respectively.

Results: The results showed that the implementation of the booklet favors more lasting thoughts of change, with statistically significant differences between the groups in moment two $(p=0.020)$ and three $(p=0.003)$ and between moments zero and one $(p<0.0001)$, moments zero and two $(p=0.001)$, moments zero and three $(p<0.0001)$ and moment two and three $(p<0.0001)$ in the group intervention.

Conclusion: The educational booklet was therefore validated and favored the advance in the stage of readiness for behavior change, proving to be effective for educational health practices. The validation of the booklet allows its use in health education activities reliably in the public in question. 


\section{Resumen}

Objetivo: Poner a prueba la efectividad de una tecnología educativa sobre exceso ponderal respecto al nivel de prontitud para cambios de comportamiento de adultos con hipertensión arterial sistémica y exceso ponderal.

Métodos: Estudio cuasi experimental con dos grupos, uno de comparación y otro experimental, cada uno con 36 participantes, cuyo nivel de prontitud para cambios de comportamiento fue evaluado antes (momento cero) y después (momento uno) de la intervención (implementación o no de la cartilla), después de un mes (momento dos) y dos meses después (momento tres) del primer contacto, por teléfono. El nivel de prontitud para cambios de comportamiento se comparó antes y después para muestreo en pares y entre los grupos para grupos independientes mediante el test-T de Student.

Resultados: Los resultados demostraron que la implementación de la cartilla favorece pensamientos de cambios de mayor duración, con diferencias estadísticamente significativas entre los grupos en el momento dos $(p=0,020)$ y tres $(p=0,003)$ y entre los momentos cero y uno ( $p<0,0001)$, momentos cero y dos $(p=0,001)$, momentos cero y tres $(p<0,0001)$ y momento dos y tres $(p<0,0001)$ en el grupo experimental.

Conclusión: Por lo tanto, la cartilla educativa fue validada y favoreció el avance del nivel de prontitud para cambios de comportamiento, por lo que se mostró efectiva para prácticas educativas en salud. La validación de la cartilla permite que sea utilizada en actividades de educación en salud de forma confiable para el público en cuestión.

\section{Introdução}

Comportando-se como doença crônica e como fator de risco para outras doenças crônicas não transmissíveis (DCNT), a obesidade apresenta dados epidemiológicos crescentes no Brasil. Um em cada cinco brasileiros têm excesso ponderal (EP). Ao longo de dez anos, a prevalência passou de $11,8 \%$ para $18,9 \%$. Esse aumento contribui para o aumento da diabetes e da hipertensão e favorece complicaçôes cardiovasculares, sobretudo diante da associação de duas ou mais DCNT. A hipertensão arterial sistêmica (HAS) também apresentou aumento de 22,5\% para $25,7 \%$ no mesmo período e país. ${ }^{(1)}$

Apesar de a obesidade e suas possibilidades de tratamento serem amplamente divulgadas, ainda existem poucos estudos de intervenção sobre a intenção de mudança dos comportamentos de risco, sobretudo entre aqueles com HAS e EP associados, colocando mais em discussáo o controle da massa corporal. Desta forma, como parte da terapêutica, faz-se relevante avaliar como o indivíduo se sente frente à possibilidade de mudança de comportamento e como proporcionar a motivaçáo para a mudança de estilo de vida, refletindo nos resultados esperados com condutas mais saudáveis. ${ }^{(2)}$

De acordo com o modelo transteorético, ${ }^{(3)}$ também conhecido como modelo dos estágios de prontidão para mudança de comportamento, as pessoas passam por diferentes estágios de motivação para a modificação de um comportamento considerado problema. O estágio de mudança reflete diretamente sobre o quanto o indivíduo está motivado efetivamente a mudar, ou seja, a disposição para aderir à mudança. Os estágios incluem: pré-contemplação, contemplação, preparação, ação e manutenção. ${ }^{(3)}$ Identificar o estágio é importante para escolher estratégias terapêuticas adicionais de motivação, com o intuito de favorecer mudança de comportamento.

Neste ínterim, desenvolveu-se uma cartilha educativa sobre EP no adulto com HAS validada quanto a conteúdo por especialistas docentes (índice de validação de conteúdo igual a 0,78 ) e quanto à aparência por especialistas assistenciais, de design e com o público-alvo (índice de concordância superior a $80 \%$ ), sendo considerada adequada. ${ }^{(4)}$ A cartilha foi intitulada "Alimentação e atividade física no adulto hipertenso e acima do peso: disposto a mudar?" e, segundo as autoras, ${ }^{(4)}$ é complementar à prática de educação em saúde para uma população que vem crescendo, haja vista o aumento das Doenças Crônicas Não Transmissíveis em nível mundial, sendo sugerida a sua validação clínica.

Além dessa perspectiva, a tecnologia foi desenvolvida levando em consideração o estágio de mudança de comportamento. Enquanto muitas intervençôes já disponibilizadas na literatura usam a linguagem imperativa, esta cartilha trabalha a perspectiva diferencial de o indivíduo se identificar com o personagem, aproximando-o das possibilidades de mudança, em vez de afastá-lo. Dessa forma, também pode ser uma ferramenta de aproximação do profissional de saúde com o paciente, vínculo essencial nesse processo de prontidão para mudança.

A partir desse contexto, objetivou-se testar a efetividade de uma tecnologia educativa sobre EP no estágio de prontidão para mudança de comportamento (EPMC) do adulto com HAS e EP. 


\section{Métodos}

Tratou-se de estudo quase experimental, realizado com dois grupos: comparação (grupo A) e intervenção (grupo B). A intervenção constituiu na aplicação da cartilha educativa à população de adultos com HAS e EP. O estudo foi realizado em um ambulatório especializado, referência em prevenção e tratamento de complicaçóes de pacientes com diabetes e hipertensão no Estado do Ceará.

A população do estudo foi composta por todos os adultos com HAS e EP devidamente cadastrados e acompanhados no lócus do estudo. A amostra do estudo foi calculada a partir da fórmula para estudos com grupos comparativos, conforme descrito a seguir: $n=(p 1 \cdot q 1+p 2 \cdot q 2) \cdot\left(Z_{\alpha / 2}+Z_{\beta}\right)^{2} /(p 1-p 2),{ }^{(5)}$ onde: $\mathrm{n}=$ tamanho da amostra para cada grupo; p1 = porcentagem estimada de adultos do grupo A com estágio de prontidão para mudança no comportamento no nível preparação $(\mathrm{p} 1=0,50)$; $\mathrm{q} 1=$ complementar de p1 (q1=0,50); $\mathrm{p} 2=$ porcentagem estimada de adultos do grupo B com estágio de prontidão para mudança no comportamento no nível preparação $(\mathrm{p} 2=0,80) ; \mathrm{q} 2=$ complementar de $\mathrm{p} 2$ $(q 2=0,20) ; Z_{\alpha / 2}=$ nível de significância fixado $(1,96)$ e $Z_{\beta}=$ poder do teste fixado $(0,84)$.

Assim, a amostra para cada grupo foi inicialmente calculada com 36 adultos, com distribuição similar quanto à faixa etária (20 a 59 anos), com HAS e EP, devidamente cadastrados e em acompanhamento, que aceitaram participar da pesquisa e estavam presentes no local do estudo durante os dias de coleta, configurando-se esses itens como critérios de seleção. Ressalta-se que a faixa etária selecionada foi estabelecida, levando em consideração a idade mínima dos cadastrados adultos no serviço que era de 20 anos e, o limite superior de 59 anos, estabelecido para náo incluir idosos, categoria com peculiaridades que poderiam gerar viés de análise e, consequentemente, no efeito da intervenção.

Os participantes foram selecionados por conveniência e, ao estarem presentes nos dias de coleta, iam sendo alocados para grupo intervenção ou comparação alternadamente. Destaca-se a dificuldade para o alcance da amostra, pois a maioria dos pacientes do serviço era idoso. Foram excluídos do estudo aqueles com déficit cognitivo que inviabilizasse a compreensão do material, bem como os que possuíam condiçôes clínicas que interferissem no EP, tais como distúrbios endócrinos e gestação. ${ }^{(6)}$ Todos esses critérios de exclusão foram identificados a partir da consulta ao prontuário. Os critérios de descontinuidade incluíram: adoecimento ou falecimento, não localização de endereço e número de telefone inexistente ou que não atende.

Foram convidados todos os pacientes que atendiam aos critérios até que se chegasse ao valor calculado para a amostra. Ao final, foi possível compor uma amostra de 33 participantes para o grupo intervenção e 34 participantes para o grupo comparação, já que, no grupo comparação, dois participantes adoeceram e estavam internados e, no grupo intervenção, dois participantes não atenderam ao telefone e um desistiu de continuar. Conforme cálculo da amostra, 36 pacientes foram incluídos no estudo, no entanto, devido aos critérios de descontinuidade, esse quantitativo foi reduzido ao final da coleta.

A intervenção a ser avaliada pelo estudo consistiu na aplicação de uma cartilha intitulada "Alimentação e atividade física no adulto com hipertensão e acima do peso: disposto a mudar?", composta por 32 páginas, formato livreto, com folha A4 dobrada ao meio, impressa em papel couchê e encadernada, com capa, apresentação, ficha catalográfica, sumário e os itens: I - Meu excesso de peso influencia na minha pressão?; II - Quais são as vantagens de mudar o meu estilo de vida?; III - Será que me alimento direito?; IV - Preciso fazer atividade física? Meu espaço, Anotações e Referências, além da mensagem no verso: "Mais importante que a vontade de mudar é a coragem de começar". Essa tecnologia mostrou-se válida em conteúdo e aparência, sendo indicada para validaçóes clínicas em processos educativos em saúde. ${ }^{(4)}$

No dia estabelecido para a pesquisa, os participantes do grupo intervençáo eram identificados e a cartilha era disponibilizada para que eles a lessem (sem intervenção inicial da pesquisadora), cujo tempo de leitura variou de dez a 20 minutos. Ressaltase que apenas um participante não sabia ler, mas as imagens eram autoexplicativas (característica do 
tipo de tecnologia). Aqueles que tiveram dúvidas foram esclarecidos pela pesquisadora à medida que a visualização ou leitura acontecia. Ao finalizar esse processo, a cartilha era gentilmente recolhida pela pesquisadora para utilização em outros momentos.

A partir da primeira leitura da cartilha, nova intervenção foi realizada após um mês, a qual consistiu na entrega de um exemplar da cartilha pela pesquisadora por meio da realizaçáo de uma visita domiciliária ao paciente para que ele pudesse fazer sucessivas leituras em casa. Destaca-se que a visita foi realizada apenas como meio de entrega da tecnologia, visto que, no primeiro momento da intervenção, ainda no serviço de saúde, as cartilhas foram recolhidas. Assim, nenhuma outra intervenção foi realizada pela pesquisadora no momento da visita.

O grupo comparação não foi submetido a qualquer tipo de intervençáo, exceto aquela prevista na orientação educativa padrão oferecida pelo serviço, a qual aconteceu na consulta de enfermagem.

Para a coleta de dados, foram utilizados os seguintes instrumentos: formulário sobre perfil sociodemográfico e clínico desenvolvido pelos pesquisadores e instrumento de avaliaçáo do EPMC dos hábitos alimentares e de atividade física (SOC Scale). Essa fase da pesquisa foi realizada em sala apropriada de modo a garantir a privacidade do paciente, bem como em espaço tranquilo que permitisse a leitura da cartilha para aqueles que eram do grupo de intervenção. $\mathrm{O}$ período da coleta foi entre agosto de 2015 e fevereiro de 2016, considerando que nem todos os integrantes de cada grupo iniciaram a participação no mesmo dia.

A SOC Scale (Stage of change Scale) foi valida$\mathrm{da},{ }^{(7)}$ sendo traduzida e adaptada para a língua portuguesa a partir da versão original. Este sugere que, apesar das pessoas perceberem que precisam realizar mudanças em seu comportamento, elas não realizam de forma abrupta, mas sim em estágios. A escala é composta por 38 questóes, divididas em quatro domínios e as respostas apresentam cinco opçôes: 1 (pré-contemplação); 2 (contemplação); 3 (preparação); 4 (ação); e 5 (manutenção). O escore médio obtido para cada um dos domínios indica o estágio em que o indivíduo se encontra.

Os momentos em que foram avaliados os EPMC consistiram em quatro fases, onde a primeira foi an- tes da utilização da cartilha (grupo intervenção) ou consulta padrão do serviço (grupo comparação), e as demais, após a intervenção ou consulta.

$\mathrm{O}$ momento zero foi abordagem ao paciente com aplicação da SOC Scale antes da leitura da cartilha (grupo intervençáo) ou da consulta de enfermagem padrão do serviço (grupo comparaçáo). O momento 1 foi a aplicação da SOC Scale imediatamente após intervençâo educativa ou imediatamente após consulta de enfermagem. $\mathrm{O}$ momento 2 foi aplicação por telefone da SOC Scale após um mês da primeira fase. No caso do grupo intervenção, a ligaçáo telefônica aconteceu um dia após a visita domiciliária. Por fim, o momento 3 foi aplicação da SOC Scale também por telefone, em ambos os grupos, dois meses após o primeiro contato no serviço em que o estudo inicialmente foi realizado.

Ressalta-se que a coleta de dados ocorreu em dias diferentes para aqueles que compuseram o grupo comparação e o grupo intervenção e o período total de coleta envolveu os meses de agosto de 2015 a fevereiro de 2016.

Os dados presentes nos instrumentos aplicados junto à população alvo foram compilados e analisados por meio do programa estatístico IBM SPSS Statistics versão 23.0. As variáveis foram analisadas de modo descritivo, considerando frequência simples e percentual, média e desvio padrão. Os valores obtidos pré-teste e pós-teste foram comparados intra e intergrupos, utilizando-se o teste t de Student para amostras em pares e para amostras independentes. Para as variáveis numéricas, foi testada a normalidade pelo teste de Kolmogorov-Smirnov. Em todos os casos, considerou-se o nível de significância de 5\%.

A coleta iniciou após aprovação do projeto em comitê de ética, sob o CAAE No 38645414.4.0000.5534 e respeitaram-se todos os preceitos éticos e legais de pesquisa envolvendo seres humanos, conforme Resolução 466/2012 do Conselho Nacional de Saúde do Brasil.

\section{Resultados}

$\mathrm{Na}$ tabela 1, encontram-se expostos os dados sociodemográficos dos participantes dos dois grupos do estudo. 
Tabela 1. Caracterização sociodemográfica de hipertensos com excesso ponderal atendidos em um ambulatório especializado

\begin{tabular}{|c|c|c|c|c|c|}
\hline \multirow[b]{2}{*}{ Variável } & \multicolumn{2}{|c|}{ Grupo Comparação } & \multicolumn{2}{|c|}{ Grupo Intervenção } & \multirow[b]{2}{*}{$p$-value } \\
\hline & $f(\%)$ & $\begin{array}{l}\text { Min-Máx; } \\
\text { Média } \\
(\mathrm{DP})^{\dagger}\end{array}$ & $f(\%)$ & $\begin{array}{l}\text { Min-Máx; } \\
\text { Média (DP) }\end{array}$ & \\
\hline \multicolumn{6}{|l|}{ Sexo } \\
\hline Masculino & $12(33,3)$ & & $26(72,2)$ & & $0,334^{*}$ \\
\hline Feminino & $24(66,7)$ & & $10(27,8)$ & & \\
\hline \multicolumn{6}{|l|}{ Faixa etária } \\
\hline 31 a 40 anos & $3(8,3)$ & $38-59$ & $5(13,9)$ & 31-59; & $0,246^{\star \star}$ \\
\hline 41 a 50 anos & $9(25,0)$ & 51,58 & $14(38,9)$ & 49,33 & \\
\hline 51 a 59 anos & $24(66,7)$ & $(+6,31)$ & $17(47,2)$ & $(+7,55)$ & \\
\hline \multicolumn{6}{|l|}{ Escolaridade } \\
\hline Analfabeto/Fundamental & $22(61,1)$ & & $12(30,6)$ & & $0,051^{* *}$ \\
\hline Médio & $11(30,6)$ & & $19(52,8)$ & & \\
\hline Técnico/Superior & $3(8,3)$ & & $6(16,7)$ & & \\
\hline \multicolumn{6}{|l|}{$\begin{array}{l}\text { Tempo de } \\
\text { acompanhamento no } \\
\text { serviço }\end{array}$} \\
\hline 1 a 5 anos & $8(22,2)$ & $1-27$ & $6(16,7)$ & & $0,013^{*}$ \\
\hline 6 a 10 anos & $9(25,0)$ & 12,39 & $21(58,3)$ & $\begin{array}{c}1-25 \\
9,36(+4,90)\end{array}$ & \\
\hline 11 ou mais anos & $19(52,8)$ & $(+7,50)$ & $9(25,0)$ & & \\
\hline \multicolumn{6}{|l|}{ Raça } \\
\hline Branca & $18(50,0)$ & & $14(38,9)$ & & $0,343^{*}$ \\
\hline Não branca & $18(50,0)$ & & $22(61,1)$ & & \\
\hline \multicolumn{6}{|l|}{ Estado civil } \\
\hline Solteiro & $6(16,7)$ & & $5(13,9)$ & & $0,742^{\star \star}$ \\
\hline Casado & $22(61,1)$ & & $26(72,2)$ & & \\
\hline Viúvo & $4(11,1)$ & & $2(5,6)$ & & \\
\hline Divorciado/separado & $4(11,1)$ & & $3(8,3)$ & & \\
\hline \multicolumn{6}{|l|}{ Renda familiar } \\
\hline Menos de $1 \mathrm{SM}^{\ddagger}$ & $1(2,8)$ & 300-6000; & $1(2,8)$ & 780-10000; & $0,113^{\star \star}$ \\
\hline 1 a $3 \mathrm{SM}^{\ddagger}$ & $34(94,4)$ & 1561,56 & $29(80,6)$ & 2345,00 & \\
\hline $4 \mathrm{SM}^{\ddagger}$ ou mais & $1(2,8)$ & $(+997,61)$ & $6(16,7)$ & $(+1971,50)$ & \\
\hline
\end{tabular}

†Desvio-padrão; ${ }^{\dagger}$ Salário Mínimo; *teste qui-quadrado de Pearson; **teste razão de verossimilhança.

Verificou-se predominância do sexo feminino no grupo comparação e no grupo intervenção, houve predominância do sexo masculino. A idade nos dois grupos variou de 31 a 59 anos, com média de 51,58 (+6,31) no grupo comparação e 49,33 $(+7,55)$ no grupo intervenção. A faixa etária predominante foi de 51 a 59 anos para ambos os grupos. A escolaridade variou de analfabeto a nível superior, com predominância do ensino fundamental completo no grupo comparação e do ensino médio no grupo intervenção. Apenas um adulto relatou náo saber ler e escrever e um referiu ter ensino superior completo. O tempo de acompanhamento dos pacientes participantes no ambulatório especializado foi maior para o grupo comparação em relação ao grupo intervenção $(p=0,013)$.

Em relação à raça, metade do grupo comparação se considerou náo branca, enquanto esse valor subiu para $61,1 \%$ no grupo intervenção. A renda familiar também foi investigada. Esta variou de R\$ 300,00 a $\mathrm{R} \$ 6.000,00$ no grupo comparação e de $\mathrm{R} \$ 780,00$ a $\mathrm{R} \$ 10.000,00$ no grupo intervenção. A maioria referiu ganhar de 1 a 3 salários mínimos, em ambos os grupos, considerando o valor do salário mínimo vigente no período da coleta, que era de $\mathrm{R} \$ 788,00$. O estado civil de mais da metade $(61,1 \%)$ do grupo comparação e da maioria $(72,2 \%)$ do grupo intervenção era casado. Os participantes também foram interrogados sobre com quem residiam. A resposta mais frequente foi residir com companheiro (a) e filho(s). A partir dessa caracterização, observa-se uma semelhança na maioria das características analisadas.

Após a caracterização sociodemográfica, estão apresentados na tabela 2 os resultados da avaliação do EPMC em cada um dos momentos da validação clínica, por grupo.

Tabela 2. Avaliação do estágio de mudança de comportamento em cada um dos momentos da validação clínica, de acordo com o grupo comparação e intervenção

\begin{tabular}{|c|c|c|c|}
\hline Avaliação do estágio & $\begin{array}{c}\text { Grupo } \\
\text { comparação } \\
f(\%)\end{array}$ & $\begin{array}{c}\text { Grupo } \\
\text { Intervenção } \\
f(\%)\end{array}$ & Teste t \\
\hline \multicolumn{4}{|l|}{ Momento 0 (linha de base) } \\
\hline Pré-contemplação & $0(0,0)$ & $1(2,8)$ & \\
\hline Contemplação & $7(19,4)$ & $6(16,7)$ & \\
\hline Preparação & $17(47,2)$ & $13(36,1)$ & \\
\hline Ação & $10(27,8)$ & $16(44,4)$ & \\
\hline Manutenção & $2(5,6)$ & $0(0,0)$ & \\
\hline Média (desvio-padrão) & $3,23( \pm 0,73)$ & $3,16( \pm 0,74)$ & $p=0,887$ \\
\hline \multicolumn{4}{|l|}{ Momento 1} \\
\hline Pré-contemplação & $0(0,0)$ & $0(0,0)$ & \\
\hline Contemplaç̧̃̃o & $3(8,3)$ & $4(11,1)$ & \\
\hline Preparação & $20(5,6)$ & $10(27,8)$ & \\
\hline Ação & $10(27,8)$ & $21(58,3)$ & \\
\hline Manutenção & $3(8,3)$ & $1(2,8)$ & \\
\hline Média (desvio-padrão) & $3,38( \pm 0,64)$ & $3,48( \pm 0,63)$ & $p=0,348$ \\
\hline \multicolumn{4}{|l|}{ Momento 2} \\
\hline Pré-contemplação & $0(0,0)$ & $0(0,0)$ & \\
\hline Contemplaç̧ão & $4(11,8)$ & $2(5,7)$ & \\
\hline Preparação & $19(55,9)$ & $12(34,3)$ & \\
\hline Ação & $10(29,4)$ & $17(48,6)$ & \\
\hline Manutenção & $1(2,9)$ & $4(11,4)$ & \\
\hline Média (desvio-padrão) & $3,16( \pm 0,68)$ & $3,63( \pm 0,62)$ & $p=0,020^{*}$ \\
\hline \multicolumn{4}{|l|}{ Momento 3} \\
\hline Pré-contemplação & $1(2,9)$ & $0(0,0)$ & \\
\hline Contemplação & $2(5,9)$ & $1(3,0)$ & \\
\hline Preparação & $21(61,8)$ & $10(30,3)$ & \\
\hline Ação & $9(26,5)$ & $19(57,6)$ & \\
\hline Manutenção & $1(2,9)$ & $3(9,1)$ & \\
\hline Média (desvio-padrão) & $3,21( \pm 0,70)$ & $3,75( \pm 0,56)$ & $p=0,003^{*}$ \\
\hline
\end{tabular}

${ }^{*} p$-value significativo para teste $t$ de Student para amostras independentes 
$\mathrm{Na}$ linha de base, em relação aos participantes do grupo comparação, demonstrou-se que eles se encontravam distribuídos nos estágios de contemplação até estágio de manutenção, com maior frequência no estágio preparação $(47,2 \%)$, ou seja, está definindo planos para mudar dentro de um mês. Já o grupo intervençáo, na linha de base, apresentou maior frequência de pacientes no estágio ação $(44,4 \%)$, ou seja, começou a mudança de comportamento nos últimos seis meses. Neste momento da segunda fase, não houve diferença significativa nas médias entre os dois grupos ( $\mathrm{p}=0,887)$. No momento 1 , também não houve diferença significativa nas médias entre os dois grupos $(\mathrm{p}=0,348)$.

No momento seguinte, que aconteceu após um mês do primeiro encontro com os participantes, ocorreu a entrega da cartilha por meio da visita domiciliário para os integrantes do grupo intervençáo e foi aplicada a escala por telefone no dia posterior. É possível visualizar na tabela que as frequências nos estágios de prontidão para mudança de comportamento do grupo intervenção foram mais evidentes que no grupo comparação, ressaltadas pelo teste de diferenças de médias entre os grupos que se apresentou estatisticamente significante $(\mathrm{p}=0,020)$.

No terceiro momento, foi possível visualizar a continuação no aumento das frequências no estágio "ação" do grupo intervenção, em detrimento do reaparecimento de frequência no estágio "pré-contemplação" do grupo comparação. As diferenças de médias também foram significantes $(\mathrm{p}=0,003)$.

Após verificação de diferença de médias entre os grupos comparação e intervenção em cada momento da fase de validação clínica, procedeu-se à análise entre etapas, para cada grupo. Os resultados são visualizados na tabela 3 .
Conforme é possível perceber na tabela 3, na comparação das médias da linha de base e do momento um, houve diferença estatisticamente significativa para ambos os grupos $(\mathrm{p}=0,012$ para o grupo comparaçáo e $\mathrm{p}=0,000$ para o grupo intervenção). Isto se deve ao fato de que intervençôes educativas e orientaçôes podem ter impacto imediato sobre o estágio de prontidão para mudança de comportamento do indivíduo. Diante da orientação, o paciente se sensibiliza e pensa em mudar. Entretanto, não é possível saber por quanto tempo o pensamento de mudança permanecerá ativo.

Na comparação de médias da linha de base e do momento dois, a diferença só foi estatisticamente significativa para o grupo intervenção $(p=0,001)$, o que sugere que a intervenção educativa no formato de cartilha promoveu pensamentos de mudança mais duradouros que a orientação oferecida durante uma consulta ambulatorial de enfermagem $(p=0,624)$. Percebeu-se também diferença do momento dois em relação ao três no grupo intervenção $(\mathrm{p}=0,000)$, reforçando a característica do efeito a curto prazo.

\section{Discussão}

Com a intervenção realizada, verificou-se a alteração no estágio de prontidão para mudança no grupo de adultos em questão, cujas características sociodemográficas eram semelhantes, exceto para o tempo de acompanhamento. No entanto, depreende-se que não houve interferência nos resultados da intervenção devido a essa diferença, pois é esperado que o tempo maior de acompanhamento sensibilize mais os pacientes e os motive mais para a mudança,

Tabela 3. Comparação entre 0 efeito da consulta ambulatorial de enfermagem e 0 uso da cartilha educativa a curto e médio prazo no escore do estágio de mudança de comportamento

\begin{tabular}{|c|c|c|c|c|c|c|}
\hline \multirow{3}{*}{ Momentos } & \multicolumn{6}{|c|}{ Grupo } \\
\hline & \multicolumn{3}{|c|}{ Comparação $^{\dagger}$} & \multicolumn{3}{|c|}{ Intervençãã $0^{\ddagger}$} \\
\hline & Média (desvio-padrão) & Teste $\mathrm{t}$ & p-value & Média (desvio-padrão) & Teste $\mathrm{t}$ & $p$-value \\
\hline $0-1$ & $3,23( \pm 0,73)-3,38( \pm 0,64)$ & $-2,646$ & $0,012^{\star}$ & $3,16( \pm 0,74)-3,48( \pm 0,63)$ & $-3,924$ & $0,000^{*}$ \\
\hline $0-2$ & $3,23( \pm 0,73)-3,16( \pm 0,68)$ & $-0,494$ & 0,624 & $3,16( \pm 0,74)-3,63( \pm 0,62)$ & $-3,648$ & $0,001^{*}$ \\
\hline $0-3$ & $3,23( \pm 0,73)-3,21( \pm 0,70)$ & $-0,197$ & 0,845 & $3,16( \pm 0,74)-3,75( \pm 0,56)$ & $-3,909$ & $0,000^{*}$ \\
\hline $1-2$ & $3,38( \pm 0,64)-3,16( \pm 0,68)$ & 1,071 & 0,292 & $3,48( \pm 0,63)-3,63( \pm 0,62)$ & $-1,221$ & 0,230 \\
\hline $1-3$ & $3,38( \pm 0,64)-3,21( \pm 0,70)$ & 1,044 & 0,304 & $3,48( \pm 0,63)-3,75( \pm 0,56)$ & $-1,530$ & 0,136 \\
\hline $2-3$ & $3,16(+0,68)-3,21( \pm 0,70)$ & $-0,667$ & 0,509 & $3,63(+0,62)-3,75( \pm 0,56)$ & $-4,867$ & $0,000^{*}$ \\
\hline
\end{tabular}

tutilizou consulta ambulatorial de enfermagem e ligações; ‘utilizou cartilha educativa, visita domiciliar e ligação; ${ }^{*} p$-value significativo para teste t de Student para amostra em pares 
pois tem maior contato com as orientaçóes. Como visto, apesar do menor tempo de acompanhamento, houve uma maior mudança de comportamento no grupo da intervenção quando comparado ao grupo controle. Reitera-se que esses grupos devem ter características homogêneas para que se possa atribuir a mudança de comportamento à intervenção e não a outros fatores.

A alteração no estágio de prontidão é um fato significativo, pois sabe-se que embora a enfermidade acometa mais os idosos, pessoas jovens têm desenvolvido a doença cada vez mais cedo em virtude do consumo de uma alimentação rica em sal e gordura e pobre em frutas, vegetais e legumes, devido ao aumento do peso e do sedentarismo. ${ }^{(8,9)}$ Assim, estão em risco para outras doenças cardiovasculares de forma prematura ${ }^{(10)}$ e, consequentemente, precisam ser alvo de intervençôes de promoção da saúde por parte dos profissionais de saúde.

A identificação dos EPMC para hábitos alimentares e atividade física em uma população pode ser considerada um dos passos iniciais no processo de desenvolvimento de estratégias de intervenção, pois, para cada comportamento encontrado, esforços específicos podem ser empregados.

Estudos que utilizaram este modelo para subsidiar políticas de promoçáo da atividade física e perda de peso na comunidade e serviços de saúde obtiveram sucesso e podem servir de exemplos para outras intervençóes. No entanto, parte dos indivíduos que procuram serviços de saúde para mudança de comportamento não o faz com a motivaçáo necessária, sendo o aconselhamento profissional, a partir da utilização de tecnologias, capaz de aumentar tal motivação e, consequentemente, as chances de sucesso. ${ }^{(11,12)}$

A avaliação do estágio de mudança de comportamento permite, portanto, classificar os indivíduos em seus respectivos estágios, possibilitando distinguir aqueles que realmente estão dispostos a modificar seu estilo de vida daqueles que não desejam mudar. É importante destacar que as intervençōes tendem a ser mais eficientes naqueles que estão dispostos a mudar seu comportamento. ${ }^{(13,14)}$

Além disso, ressalta-se que se o aconselhamento profissional quanto à importância da mudança de comportamento ocorre antes que patologias se instalem, indivíduos mais motivados podem procurar tratamento e evitar complicaçóes. Ainda que alguns indivíduos náo estejam prontos para o tratamento, cabe aos profissionais de saúde o aconselhamento, baseado não somente em informaçóes de saúde, mas também em estratégias que visem à motivação para as mudanças, como no caso da cartilha validada. ${ }^{(4)}$

Não há ensaios clínicos ou estudos quase-experimentais que utilizaram uma intervenção educativa impressa para avaliar EPM. No entanto, em estudo observacional, diferente dos achados aqui expostos, quase metade dos participantes de outro estudo que investigou o estágio motivacional estava no estágio contemplação, indicando que sabem do problema, estão dispostos a superá-lo, mas ainda não estão totalmente comprometidos com a decisão. ${ }^{(11)}$

Isso significa que eles sabem dos benefícios de modificar o estilo de vida, reconhecem sua importância para a saúde, mas há barreiras que dificultam ou impedem sua adesão como aquelas: relacionadas ao indivíduo - déficit cognitivo, baixa escolaridade, sentimentos de incapacidade, alcoolismo, socioeconômicos, aceitação da doença e esquecimento; ao tratamento - alto custo dos medicamentos, longa duração e complexidade do tratamento, efeitos adversos e número de medicamentos; e à doença complicações tardias, assintomatologia, condições da doença e cronicidade, serviços de saúde, insuficiência de informação, dificuldades no acesso e habilidade deficiente dos profissionais para ensinar $\mathrm{o}$ uso correto dos medicamentos. ${ }^{(15-17)}$

Assim, deve-se utilizar a tecnologia individualmente, já que ela tem efeito no estágio de prontidão para mudança como evidenciado nesse estudo, ou em associaçáo com outras tecnologias efetivas, ${ }^{(18,19)}$ pois sabe-se que estudos com melhores impactos na saúde cardiovascular dos participantes são aqueles que trazem a tecnologia em forma de um programa, com múltiplas estratégias envolvidas. ${ }^{(19)}$

Além de proporcionar a identificação do estágio de prontidáo para mudança de comportamento e elencar os indivíduos mais propensos a adesão da mudança do estilo de vida, a aplicação da SOC Scale apresenta-se útil para mensurar o efeito das tecnologias educativas sobre o avanço desses está- 
gios. A cartilha educativa desenvolvida e validada internamente $^{(4)}$ foi validada clinicamente neste estudo, a partir da evidência de que ela atinge ao objetivo proposto.

Algumas dificuldades foram encontradas ao longo da pesquisa, onde se destacam questóes relacionadas ao período da coleta de dados, visto que, quando esta foi iniciada, parte do corpo médico do serviço onde foi desenvolvida a pesquisa estava em gozo de férias. Esse fato reduziu significativamente a população de pacientes. Acrescentou-se a isso, a falta de medicamentos. A tensão gerada em campo, decorrente dessas vulnerabilidades institucionais, levou muitos pacientes que tinham o perfil da pesquisa a se recusarem participar da coleta ou, ainda, ocorreu de não haver pacientes com perfil para participar do estudo, levando à prorrogação do período de coleta de dados.

Entre as limitaçóes, o viés de perda de seguimento pode ter influenciado no resultado, no entanto, não ultrapassou o percentual preconizado de $30 \%$, sendo considerado mínimo. Enumera-se ainda como limitação a ausência do cegamento pelos participantes e pela pesquisadora devido à característica da tecnologia. Além disso, a pesquisadora que aplicou a intervenção foi a mesma que avaliou os efeitos nos momentos sequenciais. No entanto, todos os critérios técnicos e éticos foram rigorosamente seguidos.

Trata-se, portanto, de um material educativo voltado ao adulto hipertenso com excesso de peso e focado no estágio de prontidão para mudança de comportamento, o qual foi validado quanto ao conteúdo e aparência ${ }^{(4)}$ e, posteriormente, validado clinicamente nesse estudo, podendo ser utilizado pelo profissional de saúde na orientaçáo e acompanhamento de pacientes com esse perfil, com vistas ao avanço do estágio de prontidáo para mudança e efetiva mudança.

\section{Conclusão}

A cartilha educativa sobre EP no adulto com HAS intitulada "Alimentação e atividade física no adulto hipertenso e acima do peso: disposto a mudar?" atingiu o objetivo proposto, ao favorecer o avanço no estágio de prontidão para mudança de hábitos alimentares e prática de atividade física sendo, portanto, validada clinicamente. É uma tecnologia efetiva para o cuidado clínico do público com HAS e EP associados de perfil sociodemográfico semelhante ao desse estudo, podendo ser utilizada por profissionais de saúde como ferramenta complementar à educação em saúde.

\section{Agradecimentos}

À Coordenação de Aperfeiçoamento de Pessoal de Nível Superior (CAPES - bolsa de doutorado). Ao Conselho Nacional de Desenvolvimento Científico e Tecnológico (CNPq - edital universal).

\section{Colaborações}

Santiago JCS, Moreira TMM, Florêncio RS, Borges JWP, Pessoa VLMP e Souza ACC declaram que contribuíram com a concepçáo do estudo, análise e interpretação dos dados, redação do artigo, revisão crítica relevante do conteúdo intelectual e aprovação final da versão a ser publicada.

\section{Referências}

1. Brasil. Ministério da Saúde. Obesidade cresce $60 \%$ em dez anos no Brasil. Brasília (DF):Ministério da Saúde; 2017.

2. Stubbs RJ, Morris L, Pallister C, Horgan G, Lavin JH. Weight outcomes audit in 1.3 million adults during their first 3 months' attendance in a commercial weight management programme. BMC Public Health. 2015;15(1):882.

3. Prochaska JO, Velicer WF. The transtheoretical model of health behavior change. Am J Health Promot. 1997;12(1):38-48.

4. Santiago JC, Moreira TM. Validação de conteúdo de cartilha sobre excesso ponderal para adultos com hipertensão. Rev Bras Enferm. 2019;72(1):95-101.

5. Pocock SJ. Clinical Trials-a practical approach. Great Britiain: Wiley; 1989.

6. Brasil. Ministério da Saúde. Secretaria de Atenção à Saúde. Departamento de Atenção Básica. Estratégias para o cuidado da pessoa com doença crônica: obesidade. Brasília (DF): Ministério da Saúde; 2014.

7. Cattai GB, Hintze LJ, Nardo N Junior. Validação interna do questionário de estágio de prontidão para mudança do comportamento alimentar e da atividade física. Rev Paul Ped 2010;28(2):194-99. 
8. Malachias MV, Souza WK, Plavnik FL, Rodrigues Cl, Brandão AA, Neves MF, et al. $7^{a}$ Diretriz Brasileira de Hipertensão Arterial. Arq Bras Cardiol. 2016;107(3 Supl.3):1-83.

9. Abebe S, Yallew WW. Prevalence of hypertension among adult outpatient clients in hospitals and its associated factors In Addis Ababa, Ethiopia: a hospital based cross-sectional study. BMC Res Notes. 2019;12(1):87.

10. Vasan RS. High blood pressure in young adulthood and risk of premature cardiovascular disease: calibrating treatment benefits to potential harm. JAMA. 2018;320(17):1760-3.

11. Cunha DB, Souza BS, Veiga GV, Pereira RA, Sichieri R. Prontidão para mudança de comportamento e variação do consumo alimentar de adolescentes participantes de ensaio comunitário de base escolar em Duque de Caxias, RJ. Rev Bras Epidemiol. 2015;18(3):655-65.

12. Leão JM, Lisboa LC, Pereira MA, Lima LF, Lacerda KC, Elias MA, etal. Estágios motivacionais para mudança de comportamento em indivíduos que iniciam tratamento para perda de peso. J Bras Psiquiatr. 2015;64(2):107-14.

13. Abdi J, Eftekhar H, Mahmoodi M, Shojayzadeh D, Sadeghi R. physical activity status and position of governmental employees in changing stage based on the trans-theoretical model in Hamadan, Iran. Glob J Health Sci. 2015;7(5):23-32.
14. Bevilaqua CA, Pelloso SM, Marcon SS. Estágio de mudança de comportamento em mulheres de um programa multiprofissional de tratamento da obesidade. Rev Lat Am Enfermagem. 2016;24:e2809.

15. Fritzen JS, Motter FR, Paniz VM. Regular access and adherence to medications of the specialized component of pharmaceutical services. Rev Saude Publica. 2017;51:109.

16. Labata BG, Ahmed MB, Mekonen GF, Daba FB. Prevalence and predictors of self care practices among hypertensive patients at Jimma University Specialized Hospital, Southwest Ethiopia: cross-sectional study. BMC Res Notes. 2019;12(1):86.

17. Cruz LH, Pessoa MA, Farias AJ, Queiroz XS, Almeida TC. Factors related to noncompliance in the treatment of hypertension: an integrative review. Nursing (São Paulo). 2019;22(248):2560-4.

18. de Souza AC, Moreira TM, Oliveira ES, Menezes AV, Loureiro AM, Silva $\mathrm{CB}$, et al. Effectiveness of educational technology in promoting quality of life and treatment adherence in hypertensive people. PLOS One. 2016;11(11):e0165311.

19. Souza AC, Moreira TM, Borges JW. Educational technologies designed to promote cardiovascular health in adults: integrative review. Rev Esc Enferm USP. 2014;48(5):944-51. 